\title{
Les enjeux du Management stratégique en Afrique et leurs conséquences sur la formation des managers - Une étude de cas : le redressement de Dakar Marine
}

\author{
Tijane Sylla, MBA - HEC \\ PDG de Dakar Marine de 1985 à 1988 \\ Directeur Général de l'IAM
}

Ce témoignage est basé sur notre action à la tête de Dakar Marine pour réaliser son redressement et préparer sa privatisation. Depuis, notre expérience, enrichie par la gestion de plusieurs portefeuilles ministériels, par la consultation puis par la direction de l'IAM, confirme notre vision du management en contexte africain.

\section{Le défi du redressement}

Dakar Marine a été créée en 1980 après le regroupement et la restructuration de la petite et moyenne réparation navale existant au Sénégal et l'augmentation de la capacité d'accueil par l'acquisition d'un dock flottant de 60000 tonnes. La société ainsi constituée est orientée vers le marché international de la grande réparation navale; elle doit profiter de la fermeture du canal de Suez et de l'activité des grands pétroliers. Mais les prévisions de quantité de navires et d'heures de production facturées, contenues dans l'étude de faisabilité du projet, n'ont pas été atteintes, puisqu'après quatre années d'exploitation, Dakar Marine affiche:

- La perte de plus des $3 / 4$ de son capital social ;

- Un déficit cumulé de l'ordre de 3,5 milliards FCFA $^{1}$;

- Un besoin de trésorerie estimé à 683 millions FCFA en août 1985 ;

- Une baisse de $40 \%$ du plan de charge qui est passé de 1036000 à 600000 heures productives.

Sur le plan administratif, la Direction du personnel était devenue un simple service de contentieux sans pouvoir de décision dans le recrutement, dans la promotion et dans les mesures disciplinaires. D'où les lacunes observées dans la gestion des effectifs qui étaient devenus pléthoriques.

Cette situation a conduit les pouvoirs publics sénégalais, actionnaires majoritaires, à élaborer un plan de redressement que nous avons été chargés de mettre en œuvre le $1^{\text {er }}$ juin 1985. Dakar Marine compte alors 967 travailleurs, répartis en main d'œuvre directe, encadrement technique et personnels administratifs, commerciaux et de soutien logistique.

Parmi ces derniers, qui représentent près de la moitié des effectifs, figurent des cadres européens expatriés, dont les salaires représentent ceux de plusieurs dizaines d'ouvriers et employés sénégalais.

Le plan vise à assurer la survie de l'entreprise et à préparer sa privatisation. Il prévoit notamment une réduction des effectifs de $30 \%$ en vue de réaliser quelques 360 millions FCFA d'économie par an. 


\section{La vision technocratique et ses limites}

Le livre «Stratégie et structures » d'Alfred Chandler (1962) est le fondement de l'école de la Business policy d'Harvard, mais c'est Igor Ansoff (1965) qui impose l'approche formelle et rationnelle de la planification stratégique. Or la planification formalisée, qui repose sur l'analyse systématique, ne peut offrir qu'une séquence d'étapes successives représentant les scénarios du futur désiré, alors que la stratégie est un processus interactif et non la succession d'une séquence de formulation puis de mise en œuvre. Elle exige un échange continu entre l'analyse et la synthèse, entre la pensée et l'action. Mintzberg (2004), décrit ainsi le dilemme de la planification: les experts de la technostructure disposent du temps et des outils d'analyse formelle, mais ce sont les managers opérationnels qui détiennent les «données vivantes » de la réalité de l'entreprise, vécue dans les jeux de pouvoir. Il affirme aussi que la stratégie est une création qui relève plus de l'invention que du calcul.

L'ampleur des difficultés de Dakar Marine et la nécessité d'agir vite ont conduit les concepteurs du plan de redressement à opter pour une démarche de planification formalisée, s'appuyant exclusivement sur des données économiques et comptables.

Ainsi le plan de redressement a-t-il été élaboré par un groupe de travail interministériel et nous avons été nommés à la tête de Dakar Marine pour l'appliquer, selon une conception mécaniste et pyramidale qui fait descendre l'autorité et la communication du sommet de l'entreprise. Cette dichotomie entre la formulation du plan de redressement et sa mise en œuvre reproduit le clivage introduit par F.Taylor (1911) entre ceux qui conçoivent et ceux qui réalisent.

\section{Le plan de redressement a-t-il été élaboré} par un groupe de travail interministériel
Or, la situation se caractérise par une complexité et une incertitude grandissantes. $\mathrm{Du}$ point de vue économique, le marché mondial de la réparation et de la construction navales connaît une récession profonde, avec une montée du chômage. Du point de vue social, Dakar Marine est le théâtre d'une politisation accrue et d'un durcissement des relations patronales et ouvrières. Deux tendances, dont l'une affiliée au parti au pouvoir et l'autre à un parti d'opposition, se disputent le leadership du mouvement syndical.

La nécessité de tenir compte de cette réalité complexe dans toutes ses dimensions nous a conduits à modifier l'approche mécanique du plan de redressement, notamment en ce qui concerne la compression du personnel.

Les technocrates planificateurs, obnubilés par l'importance qu'ils accordent à la production de biens, négligent, voire ignorent la place capitale que la vie symbolique occupe dans les activités humaines. Or, tout système de production de biens matériels repose sur une organisation sociale qui, à son tour, renvoie à un système de représentation symbolique dont le rôle est de donner un sens à l'existence de la collectivité (Vallée L 1985).

C'est pour cette raison qu'au moment de mettre en œuvre la compression des effectifs, nous avons tenu compte des suggestions formulées par le personnel à la suite de la journée de réflexion sur les problèmes de Dakar Marine, organisée par le syndicat. Nous avons permis aux délégués du personnel d'accéder aux documents comptables de la société et donné le maximum d'informations sur la situation très critique de l'entreprise. Mais nous avons par dessus tout affirmé notre volonté de mener l'opération de compression avec équité, en préservant au maximum la 
compétence technique de l'entreprise incarnée par ses ouvriers spécialisés.

C'est ainsi que nous avons d'abord licencié des cadres expatriés puis sénégalais, supprimé des avantages accordés aux directeurs et chefs de service avant d'établir la liste des employés et ouvriers touchés par la mesure de compression. Et pour donner plus de crédibilité à notre démarche, nous avons diminué notre propre salaire. Nous n'avons pas agi ainsi, en ce qui nous concerne, pour nous poser en martyr, mais parce qu'il fallait donner l'exemple en « allant au charbon».

\section{Le management de l'entreprise africaine}

L'entreprise industrielle, en s'implantant en Afrique, a imposé ses propres normes d'organisation et de production, sans tenir compte des réalités sociales et culturelles du milieu. En effet le mode de production traditionnel, encastré dans le social et le religieux, «est anti-surplus, [car il tend] à freiner les rendements et à s'immobiliser lorsqu'il atteint [la satisfaction des besoins de subsistance] » (Sahlins M 1972). L'entreprise industrielle par contre, doit produire du surplus pour générer de la croissance économique et assurer sa reproduction. Elle s'est donc installée dans la béance qu'engendre l'écartèlement des sociétés africaines entre tradition et modernité.

Le conflit culturel qui en résulte constitue le vecteur de l'irruption de la culture ambiante dans l'organisation à travers les réseaux de communications informelles.

Des travaux de recherches en management interculturel ont tenté d'élucider les difficultés de gestion de l'entreprise africaine qui résultent de cette situation. Malheureusement, ces travaux, qui insistent davantage sur l'incompatibilité entre les cultures africaines et
Nous voulions que nos travailleurs et nos différents partenaires aient envie de suivre une direction qui n'hésite pas à donner l'exemple. $\mathrm{Ce}$ geste symbolique devait matérialiser la solidarité de tous dans le sacrifice.

C. Demers (1990) soutient que le passage de la stratégie planifiée à la stratégie réalisée nécessite toujours un ajustement. Mais nous n'avons pas seulement apporté des «ajustements dictés par l'apprentissage dans l'action ». Notre objectif était plutôt d'initier une démarche managériale qui tienne compte du système de représentation et des valeurs de nos travailleurs.

l'entreprise moderne, s'appuient sur des postulats culturalistes et behavioristes qui font des attitudes et des normes, introjectées par l'individu au cours de son éducation, les déterminants de ses perceptions et de ses comportements. La culture opèrerait comme un « logiciel mental » (Hofstede G 1991).

\section{Nous pensons que cette surestimation du déterminisme et de la cohérence de \\ l'ordre social occulte l'ambivalence des normes culturelles et engendre une surestimation de la diversité culturelle}

Nous pensons que cette surestimation du déterminisme et de la cohérence de l'ordre social occulte l'ambivalence des normes culturelles et engendre une surestimation de la diversité culturelle. En effet, Claude LéviStrauss (1958) enseigne que «l'homme est un social polymorphe », ce qui veut dire que chaque culture s'élabore à partir d'un noyau de signifiants repérables, «le capital commun », par sélection de normes culturelles acceptées et refoulement de normes contraires « mises en réserve», poursuit Georges Devreux (1970), mais «qui font aussi partie de la totalité 
culturelle» et qui peuvent réapparaître à tout moment.

Sur un autre plan, la critique persistante de la gestion des entreprises modernes, de la rationalité mécanique de cette gestion, de son utilisation abusive de la métaphore militaire (Peters T \& Waterman R, 1983), favorise la réflexion sur le développement d'un management authentiquement africain de l'entreprise.

Certes, l'entreprise africaine doit, pour être performante, se doter nécessairement de systèmes de décision, de planification et de contrôle efficaces, mais elle doit aussi bâtir son efficacité sur des valeurs partagées par les individus qui y travaillent et par les membres de la société où elle opère. Elle doit notamment, au-delà de coordination de ses activités par la standardisation des procédés et des qualifications, définir une attitude cohérente, compatible avec sa survie à long terme, face à certaines données essentielles de la culture africaine. Il s'agit pour elle de permettre aux travailleurs africains de s'approprier psychologiquement ce qu'ils appellent encore «le travail du Blanc», selon l'expression ivoirienne rapportée par $\mathrm{H}$. Bourgoin (1984).

Les insuffisances du management classique, qui utilise essentiellement l'autorité et des systèmes de contrôle bureaucratiques, face à la turbulence et à la complexité de l'environnement, ont conduit à l'élaboration du concept de culture d'entreprise, destiné à cimenter les unités, à renforcer la régulation de l'efficience et l'adaptation à l'environnement (Schein EH, 1985).

Les firmes industrielles élaborent leur culture d'entreprise en transmettant à leur personnel des valeurs issues de leur histoire, destinées à appuyer les facteurs de clés de succès de leur stratégie concurrentielle.
Mais, dans un chantier comme Dakar Marine, ces valeurs transmises risquent de demeurer un simple voile idéologique recouvrant les attitudes et les conduites des travailleurs, si un effort préalable de réconciliation avec les normes culturelles locales n'est pas entrepris. Or, comme la complexité engendrée par la rapidité des transformations sociales ne permet pas un retour intégral aux principes fondateurs de la culture traditionnelle, il faut revenir sur ces principes en considérant la totalité culturelle, c'est-à-dire l'ambivalence qui caractérise les normes et les valeurs de la société traditionnelle.

En effet considérer l'ambivalence des normes culturelles permet de comprendre ce que certains sociologues décrivent comme l'émergence progressive de nouvelles attitudes et conduites dans les sociétés africaines en transition vers la modernité.

Ces nouvelles attitudes et conduites correspondent en réalité aux «normes mises en réserve », mais actualisées par et dans les processus psychosociaux d'adaptation des individus. Le professeur de sociologie, Malick Ndiaye (1996), souligne ainsi qu'à la place de « la morale traditionnelle communautaire [...], tend à surgir un nouveau mode d'action, qui cherche à récupérer des débris de l'ancienne morale collective, pour les recycler [...] en un corps de maximes et de préceptes, tout tendu vers l'exaltation de l'individu, qui est le but de sa propre action».

Ainsi, à Dakar Marine, avons nous cherché à intégrer, dans la régulation de l'efficience, des valeurs traditionnelles «mises en réserve» derrière les comportements culturels dominants, de manière à tenir compte à la fois des changements qui ont affecté la société sénégalaise et des contraintes économiques de l'entreprise.

Nous avons, pour ce faire, adopté un style de leadership à la fois directif et consultatif car, 
en plus du besoin urgent de survie de l'entreprise, nous avions conscience que les conceptions de l'individu, du pouvoir, de la compétition et du temps que nous voulions promouvoir n'étaient pas, à priori, partagées par tous.

\section{Ce style de management offre la considé- ration individuelle et la consultation en contrepartie de l'engagement}

C'est le style de management qualifié par J.M.Burns (1978) et B.M. Bass (1997) de leadership transformationnel qui décrit le mieux notre approche. Il consiste en un processus de «persuasion des subordonnés » (Barnard, 1938), qui s'appuie à la fois sur un ressort transactionnel et un ressort transformationnel. Le ressort transactionnel repose sur la fixation d'objectifs précis, la négociation des moyens et des récompenses en fonction des résultats. Le ressort transformationnel, capital à nos yeux, repose sur le dépassement des intérêts matériels pour exprimer nos convictions, interpeller les croyances traditionnelles et mobiliser les équipes. Ce style de management offre la considération individuelle et la consultation en contrepartie de l'engagement.

Bass insiste sur les effets du leadership transformationnel mais souligne qu'il implique un mode d'utilisation du pouvoir fondé sur la gestion individualisée des subordonnés.

\subsection{L'individu et le groupe}

Assumer les décisions contenues dans le plan de redressement, les mettre en œuvre en résistant aux pressions du milieu social, tel était le problème qui se posait à nous. Les pressions du milieu social, en la circonstance, allaient bien au-delà de la réaction défensive du syndicat. En effet, nous devions, en tant que responsable, poser des actes qui bousculent les valeurs de générosité et de solidarité du milieu social.

C'était un obstacle difficile, parce qu'en Afrique noire traditionnelle, la personne humaine est un être qui ne tire sa signification que de l'appartenance à un lignage, à un clan et à une classe d'âge. L'individu, même s'il est directeur d'entreprise, n'a pas une existence autonome reconnue. Toute l'éducation, qu'il a reçue, vise à le faire participer à la vie de son groupe. On ne conçoit pas qu'il puisse prendre son propre destin en main.

Mais les changements rapides, qui accompagnent le développement et l'industrialisation, suscitent des conflits à tous les niveaux d'existence et provoquent «le retour du refoulé » (Freud), c'est-à-dire des normes sociales «mises en réserve», notamment l'émergence de l'individu.

En effet, l'école, l'entreprise et l'introduction massive de nouvelles valeurs ont engendré une rupture dans le double continuum sécurisant de l'éducation traditionnelle: continuum horizontal qui lie l'individu à tous les membres du clan, continuum vertical qui l'attache, à travers les générations du lignage, à l'ancêtre. L'individu est désormais voué à la solitude et à la compétition. Il doit s'organiser $\mathrm{du}$ dedans pour s'assurer une existence de moins en moins tributaire de sa communauté. Il est devenu sujet historique et social dans une société africaine en transition où, selon l'expression de Durkheim, «la solidarité organique », basée sur des relations contractuelles, s'est substituée à la « solidarité mécanique » communautaire. La fréquence des maladies mentales, dans les villes africaines, démontre les difficultés inhérentes à ces processus de reconstruction de la personnalité psychique.

L'entreprise, par son mode d'organisation du travail et sa conception de la responsabilité, renforce le processus d'individualisation de 
l'Africain. Aussi, par delà la maîtrise des techniques de gestion, les performances du manager africain, dépendront-elles de sa capacité à dominer le conflit qui le traverse, de sa capacité à réconcilier les objectifs de l'entreprise et les attentes de son groupe social.

«La fixation d'objectifs individuels va totalement à l'encontre de la mentalité africaine caractérisé par le souci de s'intégrer à un groupe» affirme E-M Hernandez (1997. En fait l'individu n'est pas une réalité inconnue. Car le primat de la communauté recouvre la valorisation de l'individu, de son courage physique et moral. Mieux, la société traditionnelle accepte la compétition dans le groupe de pairs pour dégager le champion, le «diambar» chez les wolofs du Sénégal. Pour être un «diambar», il faut se mobiliser, avoir de la conviction ( «fass diome» disent les wolofs). Le «diambar» est celui qui a triomphé du «Mbar », c'est à dire de la case des circoncis en tant qu'elle symbolise la classe d'âge. Il faut à ce propos rappeler que la lutte, sport roi de la société traditionnelle, n'est pas un sport collectif!

\section{« La fixation d'objectifs individuels va totalement à l'encontre de la mentalité africaine caractérisé par le souci de s'intégrer à un groupe»}

Cependant, pour dominer le conflit culturel qui l'habite, le travailleur africain, le cadre notamment, a besoin de confiance : confiance dans ses capacités professionnelles, confiance dans son supérieur hiérarchique par l'attention qu'il lui porte, confiance dans l'entreprise par la reconnaissance et par la rémunération équitable de sa contribution.

En nommant un nouveau directeur du personnel nous avons voulu non seulement améliorer le climat des relations avec le syndicat mais encore faire de la gestion de l'individu une préoccupation importante. Les nouvelles priorités ont ainsi porté sur la constitution d'une base de données des spécialités professionnelles, le diagnostic continu des besoins de formation par la hiérarchie, le suivi individualisé des stages de perfectionnement, le suivi des carrières individuelles, l'évaluation des performances individuelles par la hiérarchie et l'instauration d'une prime individuelle de rendement.

\subsection{Le pouvoir}

La structure hiérarchique pyramidale constitue une réponse au problème du partage du pouvoir dans l'entreprise industrielle classique. Cette structuration du pouvoir découle des théories mécanistes des auteurs classiques du management, notamment de F.Taylor.

Ce sont les expériences, menées par E. Mayo et son équipe, à l'usine Hawthorne, qui ont révélé l'existence des groupes informels d'appartenance et la complexité des forces psychosociales à l'œuvre dans l'organisation. Likert, continuateur de Mayo, démontre dans ses recherches que le commandement focalisé sur la tâche, la surveillance et le contrôle produit des résultats médiocres, contrairement à un commandement basé sur l'écoute et une relation de confiance.

La culture africaine considère comme normale l'inégalité de pouvoir entre les hommes. Mais, ici aussi, la source majeure d'insatisfaction concerne la façon dont les décisions sont prises et l'autorité exercée par ceux qui occupent les postes de commandement. Cela est confirmé par l'enquête menée en Côte d'Ivoire auprès de 560 cadres issus de 60 entreprises par H.Bourgoin. Les enquêtés donnent les réponses suivantes à la question portant sur le chef sous les ordre duquel ils souhaiteraient travailler: chef autocrate $2 \%$, chef paternaliste $24 \%$, chef consultatif $40 \%$ et chef démocrate $34 \%$. Il est remarquable d'observer le rejet du chef autocrate et le 
pourcentage inférieur du chef paternaliste (24\%) par rapport aux chefs consultatif et démocrate; ces deux profils de chefs obtiennent un score cumulé de $74 \%$.

S'agissant de Dakar Marine, malgré son statut d'entreprise publique, le ministre de tutelle ${ }^{2}$, qui avait sollicité avec insistance notre nomination, nous a accordé la confiance et l'autonomie nécessaires pour exécuter notre difficile mission, notamment dans son volet compression du personnel.

C'est pourquoi nous ne partageons pas les explications, souvent culturalistes, tendant à décrire le chef africain comme un autocrate despotique. Il n'y a pas une «impossible décentralisation de l'entreprise africaine », où la hiérarchie pesante imposerait la prise de la moindre décision au niveau le plus élevé, comme l'affirme trop catégoriquement professeur P. D'Irribarne.

Car, même si l'organigramme de Dakar Marine se présente sous la forme d'une structure hiérarchique par fonction, avec une direction de la production structurée selon des services techniques spécialisés, l'entreprise met sur pied des équipes ad hoc responsables de réaliser les contrats de réparation.

Il s'agit d'équipes semi autonomes comprenant un chef d'équipe et des ouvriers recrutés dans les ateliers des services spécialisés de l'entreprise. Lorsque le chantier met dans la même période quatre ou cinq navires en cale sèche, l'ambiance de travail se transforme en va et vient incessants d'ouvriers, qui se croisent entre les ateliers où les pièces sont préparées et les navires, mis au sec, où elles sont montées.

Ce mode de gestion des arrêts techniques de navires qui ramène la prise de décision et la coordination là où se trouve l'information, c'est-à-dire près des opérateurs, répond à l'impératif d'urgence qui caractérise la réparation navale.

Concernant toujours le pouvoir, l'analyse des systèmes politiques de l'Afrique précoloniale (Diagne $\mathrm{P}$, 1973) montre qu'à peu près partout, on trouvait le principe de la souveraineté collégiale. Le souverain détenait la réalité du pouvoir, mais devait, pour gouverner, faire appel aux sages, aux personnes expérimentées en vue d'obtenir un consensus. L'entreprise africaine doit conserver cette valeur essentielle et l'utiliser dans une dynamique de participation.

C'est pourquoi, de manière à favoriser la participation de travailleurs, l'élaboration et l'application $\mathrm{du}$ règlement intérieur de l'entreprise ont été précédées d'une large consultation des chefs de services et des délégués du personnel. Le syndicat fut même encouragé à tenir une assemblée générale pour informer le personnel ouvrier sur le contenu du document et sur les objectifs poursuivis par la direction. De même, des réunions périodiques sont tenues au niveau de la direction, des services et des ateliers, pour préparer les décisions et contrôler la marche de l'entreprise.

\subsection{La maîtrise de l'outil}

Les sociétés traditionnelles africaines procèdent d'une vision du monde spécifique : l'africain traditionnel se considère comme un élément de l'immense ordre cosmique que constitue l'univers. Pour l'Africain, Dieu a créé le monde par le verbe, et le verbe est création continue par le souffle de l'homme. La parole des ancêtres fonde les institutions, leur donne un sens par et dans des mythes intemporels.

Cette vision du monde est très éloignée du projet cartésien de maîtrise et de domination du monde par la Science et la Technique. 
S'agissant de l'éducation des enfants, on a d'ailleurs souvent souligné la pauvreté du milieu traditionnel africain par opposition à la richesse en éléments dits «éducatifs » dont jouit l'enfant européen. Nous pensons qu'il est plus juste de considérer que l'éducation de l'enfant africain ne vise pas à préparer son entrée dans l'univers d'images et de machines de la civilisation moderne. En effet, la proximité et le contact physique permanent avec la mère, dans la petite enfance, rendent inutile la constitution d'un espace intermédiaire peuplé d'objets à manipuler (Erny P, 1972).

La transmission de la connaissance scientifique et technique se fait de manière ésotérique à l'intérieur de castes qui sont des groupes héréditaires, endogames, à spécialisation professionnelle (Diop AB, 1981). L'apprentissage du travail artisanal se réalise soit aux côtés de l'adulte, soit au sein d'un groupe d'enfants entrainés par des aînés par l'imitation et l'identification, comme le décrit Camara Laye dans son roman autobiographique «l'Enfant Noir »: « sur un signe de mon père, les apprentis mettaient en mouvement les deux soufflets en peau de mouton, posés à même le sol, de part et d'autre de la forge [...]. Ces apprentis se tenaient constamment assis, devant les soufflets ; $[\ldots .$. l'aîné était parfois admis à partager le travail des ouvriers, mais le plus jeune ne faisait qu'observer, en attendant d'être à son tour élevé à des travaux moins rudimentaires. L'opération qui se poursuivait sous mes yeux n'était une simple fusion de l'or qu'en apparence; c'était bien autre chose: une opération magique que les génies pouvaient accorder ou refuser $;[\ldots]$ mon père remuait les lèvres! Ces paroles que nous n'entendions pas, ces paroles secrètes [étaient l'essentiel] ». Cette parole ancestrale est au cœur de la transmission ésotérique des connaissances scientifiques et techniques.
Ces réalités culturelles commandent à l'entreprise africaine d'accorder une place prioritaire à la formation technique et professionnelle du personnel ouvrier, comme les sociétés européennes l'ont fait à partir du $19^{\text {ème }}$ siècle, lorsque les corporations furent abolies.

En effet en Europe, du Moyen Age jusqu'au milieu du $18^{\text {ème }}$ siècle, les corporations regroupaient les maitres et les compagnons. Elles réglementaient et contrôlaient rigoureusement l'exercice des professions. En France, c'est seulement en $1880^{3}$ que furent créées des écoles d'apprentissage manuel. Ces structures d'apprentissage ont permis la conversion des savoir faire empiriques tacites des corporations en connaissances théoriques. Leur développement a suivi l'évolution industrielle, en s'orientant de plus en plus vers l'éclatement des métiers et des processus technologiques.

A Dakar Marine, nous avons renforcé les capacités de notre centre de formation professionnelle dont les objectifs portent essentiellement sur la formation complémentaire ouvrière, le perfectionnement technique et la préparation de la maîtrise à ses nouvelles tâches de motivation, de conseil et de contrôle de l'exécution du travail. Le centre de formation participe aussi au recrutement du personnel en procédant aux examens psychotechniques des candidats.

\section{4. Le temps}

Dans son ouvrage «Psychologie du temps » (1967), le professeur P. Fraisse soutient que le sentiment le plus primitif de durée naît, chez le nourrisson, d'une frustration d'origine temporelle. Cela veut dire que la durée ne devient une réalité psychologique que lorsque le désir n'est pas comblé dans l'immédiat, comme dans l'allaitement à des heures précises, dans les sociétés industrielles. 
Par contre, en milieu traditionnel africain, l'oralité de l'enfant se développe selon un modèle de permissivité totale et de gratification maximale jusqu'au sevrage, qui intervient vers deux ans. L'enfant, pendant toute cette période, est enveloppé dans la présence continuelle de l'ensemble des femmes $d u$ groupe familial, faite $d$ 'un allaitement à la demande et de contacts physiques permanents.

L'allaitement à la demande du nourrisson africain ne lui permettrait donc pas de développer un schème temporel en rapport avec la non satisfaction de ses désirs.

N'ayant jamais à attendre, allaité dès qu'il le demande, le nourrisson africain ne connaîtrait que des « suites labiles de maintenant » (Erny $\mathrm{P}, 1972)$.

Le temps dans la société traditionnelle se découpe en saisons cycliques et non en heures, minutes et secondes. Il est un compagnon de l'homme et non un adversaire qu'il faut battre par une innovation technique ou un effort de productivité.

\section{Le temps dans la société traditionnelle se découpe en saisons cycliques et non en heures, minutes et secondes}

Cependant, ces réalités subissent les changements profonds et rapides engendrés par l'industrialisation et l'urbanisation. Dans l'entreprise, le travail n'est plus rythmé par la succession des saisons mais par la cadence des machines et le temps quantifié du travail s'est substitué au temps qualitatif circulaire du village.

Le temps, a cause des « rongeurs de temps » que sont les pressions communautaires exigeant la présence aux nombreuses cérémonies, est une variable critique de management dans l'entreprise africaine. Sa gestion est liée à celle de l'individu.
En effet le manager africain doit faire face à l'irruption des pressions communautaires dans les réseaux de communications informelles de l'entreprise. Il doit, comme le soutient Chester Barnard (1968), gérer l'organisation informelle, tout en s'assurant que l'entreprise atteigne ses objectifs.

Aussi, est-ce pour lutter contre les « rongeurs de temps ", que nous avons limité l'accès de Dakar Marine, pendant les heures de travail, aux seules personnes disposant d'un rendez vous et réglementé les permissions accordées au personnel pour les évènements familiaux.

Pour les dirigeants africains, améliorer la gestion du temps c'est aussi déléguer des responsabilités, donner l'exemple en matière de ponctualité et de respect du temps des autres, par la gestion efficace des réunions.

Lorsque je prends fonction à la direction de l'IAM, le responsable du personnel me demande, à la fin de ma première réunion de coordination, s'il doit préparer une note de service rappelant les heures de travail. Je réponds que j'attends la ponctualité de la part de mes collaborateurs d'abord, et que leurs subordonnés suivront leur exemple. Cela s'est passé ainsi.

\subsection{Le statut de la femme}

La société africaine traditionnelle a essentiellement réservé à la femme la procréation et les occupations domestiques. Mettre au monde des enfants donne à la femme reconnaissance et statut, parce qu'elle contribue ainsi à assurer la fécondité physique du clan. La jeune fille peut être, contre son gré, donnée comme épouse et rejoindre une nouvelle famille. Il résulte de cette condition que, sur le plan de la personnalité psychique, la femme est portée à développer des sentiments latents de réserve et une attitude de repli sur son foyer et sur ses enfants. 
Mais, nous l'avons déjà souligné avec G. Devreux (1970), ce modèle culturel dominant cache celui de la femme intrépide et entrepreneur que nous décrit Hampâté Bâ ${ }^{4}$, dont l'enfance s'est déroulée dans les années 1920, à une époque où la société traditionnelle était moins déstructurée: «comme partout où elle était passée, ma mère avait réussi à développer [ ] un commerce assez important de tissus et d'articles régionaux divers».

Une conséquence importante de la modernité est de bouleverser la division sexuelle des rôles instaurée par la société traditionnelle.

Une récente étude que nous avons menée au Sénégal, pour le BIT, auprès d'un échantillon de micro et petites entreprises rurales révèle que $56 \%$ des entrepreneurs ruraux sont des hommes et $44 \%$ des femmes. Une autre enquête réalisée par les étudiants de l'IAM, sur le développement des écoles d'enseignement supérieur privé au Sénégal, révèle que 53\% des effectifs sont constitués de filles de 23 ans de moyenne d'âge et que la gestion fait partie des filières préférées.

L'entreprise africaine ne pourra pas ignorer très longtemps ces phénomènes qui transforment son environnement socioculturel. La responsabilisation des femmes dans l'entreprise libère non seulement des potentialités jusque là sous utilisées, mais encore constitue un facteur positif de changement social, car elle fait évoluer les conceptions traditionnelles de l'autorité et de la division du travail.

\section{Conclusion}

La compression des effectifs a permis de réaliser 375 millions FCFA d'économies, de payer progressivement les fournisseurs et de remonter le plan de charge grâce à la confiance retrouvée. A la fin du premier exercice, le déficit annuel de l'entreprise a été réduit de
Notre constat, après des années d'observation, est que l'entreprise africaine est gagnante en nommant des managers femmes. En effet lorsque le climat organisationnel s'y prête, les femmes affichent leur détermination à être performantes car elles veulent démontrer leur capacité à diriger. Les hommes qu'elles commandent s'investissent, car ils trouveraient honteux, selon la norme traditionnelle, que leurs supérieures femmes puissent relever des manquements dans leur travail.

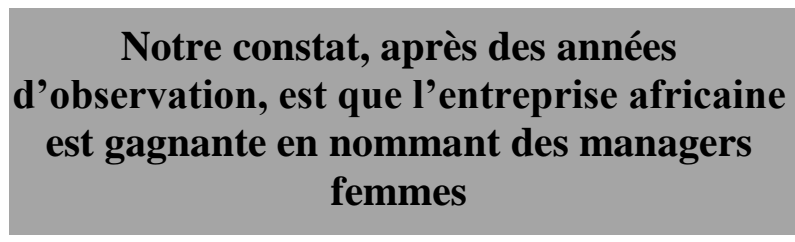

L'Institut Africain de Management (IAM) illustre cette volonté de performance des femmes. En effet, le département qui délivre le MBA, diplôme qui a reçu l'accréditation du Conseil Africain et Malgache de l'Enseignement Supérieur (CAMES) en 2007, est dirigé par une femme. Par ailleurs, l'IAM, certifié ISO 9001 version 2000, pour l'ensemble de ses processus, a nommé une femme responsable de la qualité.

A Dakar Marine, les femmes travaillaient surtout dans les services administratifs. Leur présence parmi le personnel ouvrier était extrêmement rare mais nous veillions, auprès des chefs d'ateliers, à leur participation aux activités du centre de formation pour les préparer à accéder aux responsabilités de chef d'équipe.

$60 \%$. Cependant, malgré trois années d'effort, l'équilibre financier n'est pas atteint au moment de notre départ de Dakar Marine en 1988, à cause de l'absence de recapitalisation de la société, du poids de la dette liée à 
l'acquisition du dock flottant et de la persistance de la crise.

Dakar Marine est aujourd'hui privatisée, mais beaucoup d'obstacles demeurent et l'âpreté de la concurrence dans le secteur incite à tenir compte, plus que jamais, dans les politiques à moyen terme, des conditions économiques extérieures. Mais, cette lucidité économique extérieure ne suffira pas pour assurer sa pérennité. Car le vrai défi est de bâtir une stratégie corporative qui reflète et modèle les attitudes et les valeurs des travailleurs de l'entreprise.

Il résulte des bouleversements de la société africaine que le travailleur africain ne réalise pas passivement les normes sociales de son milieu. Il est un «acteur» (Crozier M \& Friedberg E, 1977) qui utilise et transforme, dans ses interactions, les capacités culturelles acquises dans ses différents champs de socialisation que sont la famille, la société et le métier.

Il appartient aux managers africains de modeler ces valeurs, en exploitant leur ambivalence, de manière à les rendre compatibles à la fois avec les contraintes économiques de l'entreprise et les transformations de la société. Leur mission, en tant que leaders, consiste, selon le mot de Philippe Selznick (1957), à «retravailler des matériaux humains et techniques pour façonner une organisation qui met en œuvre des valeurs nouvelles et durables ».

Mais un tel projet interpelle les écoles de gestion, notamment celles opérant en Afrique. En effet, en mettant l'accent sur l'analyse quantitative et la transmission d'une boite à outils pour solutionner des problèmes prédéfinis, répertoriés dans des études de cas, les écoles de gestion classiques préparent davantage les étudiants à l'administration de choses qu'au gouvernement des hommes (Mintzberg, 2005).

Les écoles de gestion africaines ne peuvent se contenter d'être de simples centres d'apprentissage. Elles doivent plutôt former des managers responsables, des hommes d'affaires et des citoyens capables de freiner la paupérisation, de restaurer les écosystèmes, et de repositionner l'Afrique dans la division internationale du travail. Elles doivent pour cela permettre à leurs étudiants de développer leur personnalité et de réconcilier les valeurs de l'entreprise et celles de leur société.

Dans notre expérience de directeur fondateur de l'Ecole supérieure de gestion des entreprises (ESGE) ${ }^{5}$, puis de directeur général de l'Institut africain de management(IAM), nous avons accordé une place importante à la coopération internationale, mais refusé l'importation servile de programmes académiques valorisant l'élitisme, l'égoïsme et l'arrogance (Mintzberg,2005).

La philosophie d'action d'une école de gestion africaine doit plutôt s'inspirer de Socrate et d'Aristote. De Socrate, parce que sa maxime, « connais-toi toi-même », finalité de son art de la «maïeutique», est essentielle dans la formation de futurs dirigeants habités par le conflit de valeurs résultant des mutations rapides de la société africaine. D'Aristote, parce que son « réalisme des objets du monde sensible» est le fondement des sciences expérimentales. 


\section{Notes et références}

Le FCFA a été dévalué de $50 \%$ en janvier 1994 ; depuis lors $656 \mathrm{CFA}=1 €$

Serigne Lamine DIOP, Ministre de l'Industrie assurait la tutelle de Dakar Marine

Reuchlin Maurice (1971): Traité de Psychologie appliquée. PUF,

Bâ A. Hampathé: Amkoullel, l'enfant Peul. Babel 2000

ESGE : Ecole Supérieure de Gestion des Entreprises, devenue, en 1985, le CESAG

\section{Bibliographie}

- Ansoff I (1965): Stratégie de développement de l'entreprise. Ed Hommes et Technique, Paris

- Barnard C (1938): The functions of the executive. Harvard University Press, Cambridge

- Bass B.M (1997): Does the Transactional- Transformational Leadership paradigm transcend organizational and national boundaries, American psychologist, pp;130-139

- Bourgoin H (1984) : L'Afrique malade du management. Ed J. Picollec ; Paris.

- Burns J.Mc Gregor(1978): Leadership. Harper \& Row; NY.

- Chandler A (1962) : Stratégie et structures. Ed d'Organisation.

- Crozier M \& Friedberg E (1977) : L’Acteur et le Système ; Ed du Seuil ; Paris.

- Devreux G (1970) : Essais d'ethnopsychiatrie générale. Gallimard ; Paris

- Diagne P (1967) : pouvoir politique traditionnel en Afrique occidentale. Ed Présence Africaine. Diop A.B (1981): La société Wolof : tradition et changement. Ed Karthala. Paris

- Erny P (1972) : L’Enfant et son milieu en Afrique noire. Payot ; Paris

- Hafsi T, Seguin F \& Toulouse JM (2000): La stratégie des organisation:une synthèse. 2ème Ed Transcontinentale ; Montréal

- Hofstede.G(1991): Cultures and organizations. Software of the mind. Mc Graw-Hill.

- Irribarne (d') P (1999): Face à l'impossible décentralisation des entreprises africaines. Revue Française de Gestion n`80, Septembre- Octobre, P.28-39

- Levi-Strauss C (1958) : Anthropologie structurale. Plon ; Paris

- Mintzberg H (1980): The nature of managerial work. Printice Hall;NY .

- Mintzberg H (2004): Le management : voyage au centre des organisations Ed d'Organisation ; Paris

- Mintzberg H (2004): Grandeur et décadence de la planification stratégique. Dunod ; Paris

- Mintzberg H (2005): Des managers des vrais ! Pas des MBA. Ed d'Organisation ; Paris

- Ndiaye M (1996): L'éthique ceddo et la société d'accaparement ; tome1. Presse universitaire de Dakar

- $\quad$ Peters T \& Wterman R (1983): Le prix de l'excellence. Inter Edition ; Paris

- Sahlins M (1972): Age de pierre, âge d'abondance. Ed Gallimard ; Paris

- Schein E.H (1985): Organizational culture and leadership. Jossey-Bass; San Francisco

- Seguin F \& Chanlat J.F(1983): L'analyse des organisations ; Tome 1 : Ed Préfontaine, Montréal

- Selznick P (1957): Leadership in administration: a social interpretation. Harper \& Row

- Taylor F.W (1911): Principes d'organisation scientifique des usines ; Dunod, Paris

- Zadi-Kessy M (1998): Culture africaine et gestion de l'entreprise moderne. Ed Ceda, Abidjan 